Ann. Génét. Sél. anim., I979, 11 (I), I-6.

\title{
Variants colorés chez le Mouton Néerlandais des Landes de bruyère (Heideschaap)
}

\author{
J. J. LAUVERGNE et S. BOTTEMA
}

Département de Génétique animale

Centre national de Recherches zootechniques, I.N.R.A. 78350 Jouy-en-Josas (France)

Fondation Néerlandaise pour les Races Rares

Poststraat, 6, Groningue, (Pays-Bas)

\begin{abstract}
Résumé
Une première étude dans 2 troupeaux de moutons des Landes de Bruyère en Hollande, révèle un biallélisme pour les loci colorés $A, A$ gouti, $B$, Brun, E, Extension et $S$, Panachure irrégulière : $A^{\text {wh }}$ et $a$ en $A, B$ et $b$ en $B, E^{+}$et $E^{d}$ eni $E, S^{b}$ et $S^{+}$en $S$. Il y aurait peut-être aussi un autre variant de panachure et un autre facteur en $A$ gouti. Comparé à des races multivariantes comme l'Islandaise, la Corse ou le Vieux Norvégien, il s'agit d'une variabilité modérée. On pense que la variabilité fondamentale a subi une première réduction puis s'est stabilisée à son stade actuel pour une raison encore mal éclaircie.
\end{abstract}

\section{Introduction}

Il existe aux Pays-Bas un petit groupe de races ovines appelées races des Landes de Bruyère (MASON, I969). Le mot qui les désigne en hollandais est Heideschaap, littéralement, mouton des landes ou des bruyères car " heide " a cette double signification dans cette langue.

Il s'agit d'animaux qui, jadis, vivaient dans l'Est du pays sur des terrains sablonneux où la principale formation végétale était la lande de bruyère (à Calluna vulgaris) qu'ils étaient capables d'utiliser dans le cadre d'un cycle végétatif assez long avec régénération par le feu (cf. VAN BEMMEL, I963). Selon ce même auteur au début du siècle les moutons des Landes de Bruyère étaient encore très nombreux (200 00o têtes) mais, dès qu'on a commencé à faire disparaître les landes de bruyère par des procédés modernes d'amendement alors leur nombre a décru. Aujourd'hui les landes de bruyère ne sont plus qu'un souvenir et ces animaux, qui n'ont plus d'utilisation agricole aux Pays-Bas, seraient même totalement disparus si des associations pour la conservation de la nature ne maintenaient certains troupeaux. 
Actuellement il y a environ 3 ooo têtes de mouton des Landes de Bruyère répartis en 24 troupeaux disséminés dans 5 provinces et appartenant à 5 sous races: de Drenthe (Drentse Heideschaap), Schoonebeker, de Véluve (Veluwse Heideschaap), de Kempen (Kempische Heideschaap) et du Limbourg (Limburgse Heideschaap), VAN HELDEN et MiNKEMA (I978).

Le mouton des Landes de Bruyère est un mouton haut sur patte, à chanfrein modérément bombé, laine grossière, queue longue et fine, il peut ou non porter des cornes et il présente plusieurs types colorés (NUMAN I835, VAN BEMMEL I863, RYDER I974, HAZEBROEK I976), cf. figure I.

Dans le présent article nous voudrions apporter quelques précisions sur la coloration de ces races.

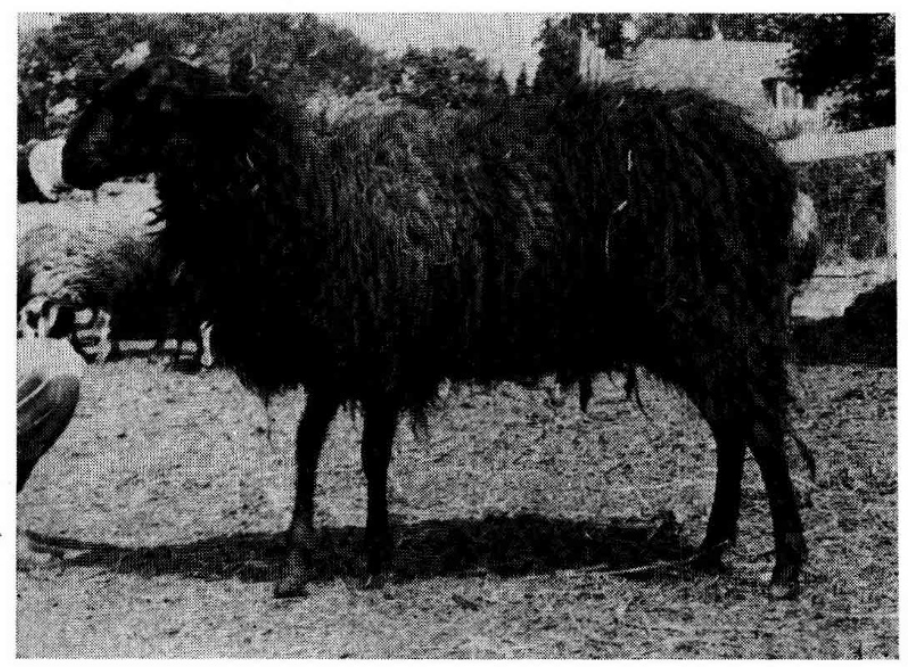

FIG. I. - Brebis Schoonebeker à Westerbork.

Schooneber ewe at Westerbork $(D R)$.

\section{Matériel et méthode}

Les animaux, tous adultes, ont été examinés en mai I978 dans 2 troupeaux communaux de la province de Drenthe :

- l'un de race Schoonebeker (environ I20 mères), sans corne, qui avait été acheté à M. NoORDHUIs, à Westerbork;

- l'autre à Ruinen, de race de Drenthe (environ 300 mères cornues ou cornettes).

I,es couleurs pigmentaires ont été mesurées avec l'atlas coloré de MǗLLER (2 54I nuances) et on les a classées à 1'aide de l'échelle colorée utilisée par la mise en évidence d'un variant brun-chocolat chez le Mouflon par DENIs et. al. (I978).

Pour les panachures on a cherché à différencier les différents types en classant des silhouettes.

En l'absence de contrôles de parenté précis (pas de monte en main), il s'est agi pour nous dans un premier temps d'isoler le maximum de phénotypes en donnant, si possible, leur correspondance génotypique. 


\section{Résultats}

\section{Les patrons pigmentaires}

A côté du blanc (absence de pigment) on a trouvé les couleurs pigmentaires suivantes :
ro-o (noir) (1)
(1), (I 2 ) 8-0,
(8) 5 -I et (8) 6-I.

Ces couleurs pigmentaires se présentaient la plupart du temps en patrons uniformes $\left(^{2}\right)$ sauf dans un cas où la face était partie noire, partie fauve, de même que les pattes, la toison étant claire mais avec des traces fauves.

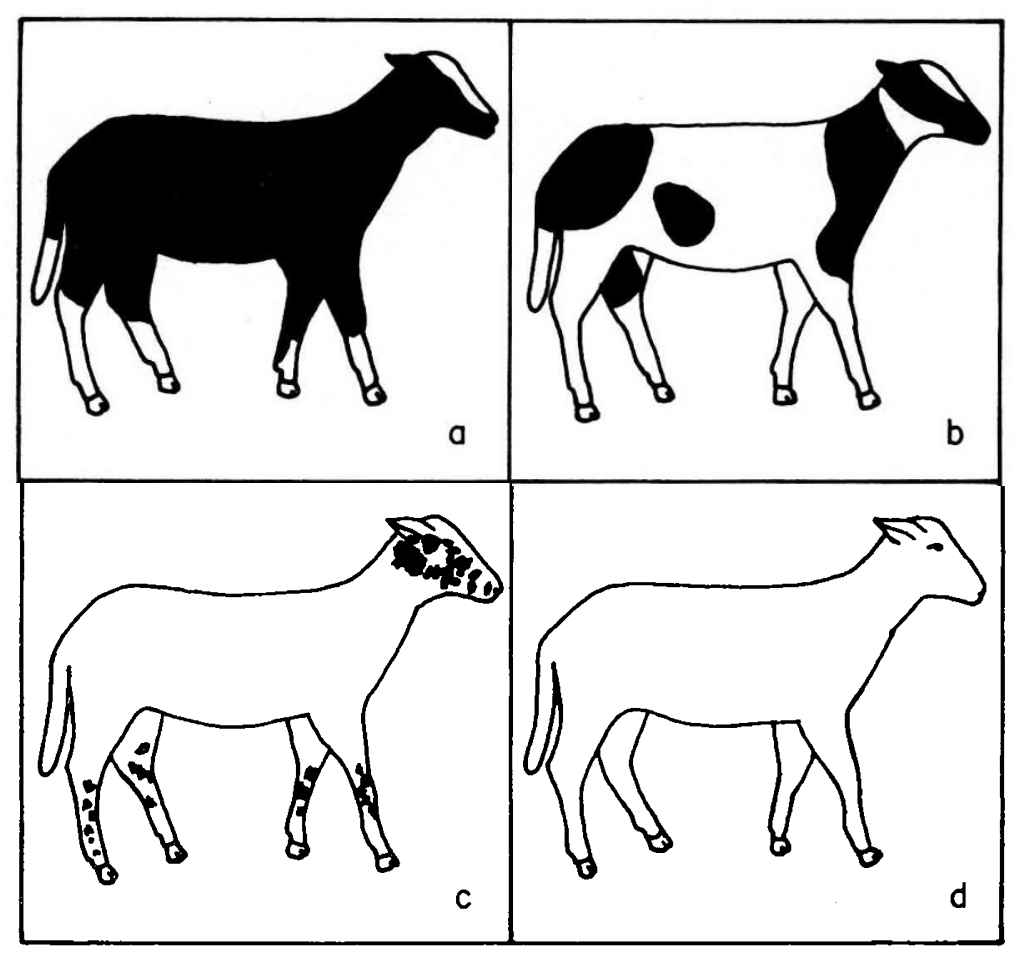

FIG. 2. - Panachures observées à Westerbork et à Ruinen.

a) Six points blancs ou HST ou patron Bizet.

b) Panachures sur le corps en plus des six points blancs.

c) Pointillés aux extrémités.

d) Tout blanc.

White designs seen at Westerbork and at Ruinen.

a) HST pattern (Head, sockings tip of tail) or Bizet pattern.

b) HS'T and piebald body.

c) Dotted extremities.

d) All white.

(1) Le chiffre entre parenthèses désigne la nuance du spectre, il est suivi par la mesure de la valeur et de la saturation, cf. DÉRIBERÉ (1964).

(2) On appelle patron pigmenté uniforme un patron où toutes les plages pigmentées 1 e sont avec un même type de pigment mélanique, on ne s'occupe pas des pages blanches dont la couleut est due à une absence de pigments. 
Selon l'échelle de DENss et al. (I978) ces colorations correspondent à de l'eumélanine noire (I0-0) à de l'eumélanine trun-chocolat [(I2) 8-0] et à de la phoemélarine fauve $[(8)$ 5-I et (8) 6-I].

Les animaux fauves sont appelés localement "vossekopen " (tête de renard). On n'est pas sûr que les éleveurs fassent bien la distinction entre le noir et le brunchocolat (svart et truin).

Souvent, chez les animaux pigmentés à la naissance (spécialement chez les fauves), la toison s'éclaircit avec 1'âge jusqu'à devenir toute blanche.

\section{Les dessins blancs}

On vient de voir que très visibles à la naissance, ils s'estompaient parfois chez l'adulte avec la dépigmentation de la toison. On a pu cependant distinguer notamment le dessin Bizet, appelé parfois HST (pour " white head, legs and tip of tail "). I1 y avait également des cas de panachure plus étendue dans lesquelles on distinguait la plupart du temps le dessin HST et aussi des cas de panachure pointillés sur la tête, en noir spécialement, et des tout blanc (cf. fig. 2).

\section{Discussion}

Les patrons pigmentaires étaient uniformes (sauf dans un cas que nous examinerons plus loin). En raisonnant par analogie avec ce qui a été vu dans les autres races (ADALSTEINSSON, I970; LAUVERGNE, I975) on peut penser tout d'abord que le rouge fauve est induit par l'allèle de tête de la série $A$ gouti: $A$ wh, la formule au locus d'Extension étant $E^{+} E^{+}$. Quant au patron uniforme eumélanique (noir ou brun-chocolat), il y a deux possibilités : soit qu'il provienne de l'allèle dominant en $E: E^{a}$ (quelle que soit alors la formule en $A$ gouti) ou alors de 1'allèle récessif en $A: a$, la formule au locus d'Extension étant alors $E^{+} E^{+}$. Des résultats de croisement sont nécessaires pour savoir si c'est l'un ou l'autre des allèles qui donne le noir ou même si tous les deux sont présents, ce qui n'est pas impossible vu qu'ils coexistent aux Pays-Bas (LaUvergne et Hoogschagen, 1978).

Le patron où, sur la tête, se juxtaposaient des mélanines noire et rouge pourrait être déterminé par un allèle apparemment non encore décrit de la série Agouti mais il aurait fallu examiner le corps chez l'animal jeune.

L'alternance noir/brun-chocolat quant à elle est attribuable à l'action d'un allèle au locus $B(B r u n)$ bien connu chez les Mammifères (SEARLE, I968), déjà mis en évidence par ADALSTEINsson (I970) chez le mouton Islandais, et plus récemment isolé chez un mouflon Corse mâle (DENIs et al., I978). Déjà RyDER (I974) avait décelé l'existence de ce mutant.

La panachure corporelle de la figure $\mathrm{I} a$ et $b$ peut être interprétée par une variation de l'expressivité de $S^{b}$ mais l'existence d'un autre facteur de panachure n'est pas à exclure, ne serait-ce que pour interpréter les pointillés de la figure Ic .

Comme les gènes $S^{b}$ et $A^{w h}$ sont présents dans la race, on peut penser que, du moins dans certains cas, le blanc uniforme est donné par l'interaction de ces deux gènes, comme chez les animaux étudiés par LAUUVERGNE (I975). Cependant on a vu récemment que pour le Texel, tout au moins (LAUvERGNE et HoogschaGEN, I978) et comme en Islande (Adalsternsson, I970), les tout blanc pouvaient être de formule $A^{w h} A^{w h} S^{+} S^{+}$. 
L'étude de la panachure est à reprendre sur les jeunes à la naissance car la canitie, surtout pour le rouge fauve et le brun-chocolat, rend rapidement les dessins blancs corporels illisibles.

Des résultats de croisements sont également nécessaires mais le peu d'espoir d'en obtenir dans le proche futur est précisément la raison pour laquelle on s'est résolu à faire cet article, avec toute son imprécision.

D'après nos examens le nombre maximum d'allèles segrégant chez le mouton des Landes de Bruyère serait donc de $8\left[E^{+} \mid E^{d}, A w h / a, B / b, S^{+} / S^{b}\right]$. On remarque que cette variation correspond (à un allèle près : $b$ absent des autres races néerlandaises) à la variation observable dans l'ensemble des populations néerlandaises " modernes" (Texel, Zwartbles, Dutch), LaUvergne et Hoogschagen (I978).

En outre on voit que cette variation correspond en gros à celle décrite par Numan (I835) qui notait déjà une proportion élevée d'animaux blancs suite à une forte demande pour les laines blanches.

\section{TABLEAU I}

Allèles des loci contrôlant la couleur du pelage

qui ont été détectés dans 6 races "primitives "de mouton en Europe

Alleles at loci controlling coat color

which have been detected in 6 "primitive" sheep breeds in Europe

\begin{tabular}{|c|c|c|c|c|c|c|c|}
\hline \multicolumn{2}{|c|}{ Variants colorés } & \multicolumn{6}{|c|}{ Races } \\
\hline Loci & Allèles & $\begin{array}{l}\text { Vieux }\left(^{(1)}\right. \\
\text { Norvégien }\end{array}$ & $\begin{array}{l}\text { Islandais } \\
{ }_{\left({ }^{2}\right)}\end{array}$ & $\begin{array}{c}\text { Faroe } \\
\left({ }^{3}\right)\end{array}$ & $\begin{array}{c}\text { Soay } \\
\left({ }^{4}\right)\end{array}$ & $\begin{array}{c}\text { Corse } \\
(5)\end{array}$ & $\begin{array}{l}\text { Landes de } \\
\text { Bruyères } \\
\left({ }^{6}\right)\end{array}$ \\
\hline$\stackrel{\text { A }}{\text { Agouti }}$ & 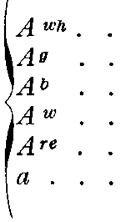 & $\begin{array}{l}+ \\
+ \\
+ \\
+ \\
+\end{array}$ & $\begin{array}{l}+ \\
\dot{t} \\
+ \\
+ \\
+ \\
+\end{array}$ & + & $\begin{array}{l}+ \\
+\end{array}$ & $\begin{array}{l}+ \\
+ \\
+ \\
+ \\
+ \\
+\end{array}$ & + \\
\hline $\begin{array}{c}\text { B } \\
\text { Brun }\end{array}$ & $\left\{\begin{array}{lll}B \cdot & \cdot \\
b \cdot & \cdot & \cdot\end{array}\right.$ & + & $\begin{array}{l}t \\
t\end{array}$ & + & $\begin{array}{l}+ \\
+\end{array}$ & + & + \\
\hline$\underset{\text { Extension }}{\mathrm{E}}$ & $\left\{\begin{array}{l}E \\
E+\end{array}\right.$ & + & + & + & + & + & $\begin{array}{l}? \\
+\end{array}$ \\
\hline $\begin{array}{c}\mathrm{S} \\
\text { Panachure } \\
\text { irrégulière }\end{array}$ & $\begin{cases}S^{+} & \cdot \\
S^{b} \cdot & \cdot\end{cases}$ & + & + & + & + & + & $\stackrel{+}{+}$ \\
\hline
\end{tabular}

( $\left.{ }^{1}\right)$ Interprétation allélique des données de BERGE (1958).

(2) ADALSTEINSSON (1970).

$\left({ }^{3}\right)$ Adalsteinsson et Wardum (r978).

(4) RYDER et al. (I974).

(5) IAUUVERGNe et ADALSTEINSSON (I976).

(6) Présent article. 
En fait, comparée aux variabilités enregistrées dans certaines autres races européennes considérées aussi comme primitives la variabilité en races des Landes de Bruyère est relativement réduite (cf. tab1. I).

Toutefois, le prccessus de blanchiement qui, après avoir touché d'autres races européennes semblait avoir atteint les races des Landes de Bruyère dès le début $d u X{ }^{e}{ }^{e}$ siècle, se serait interrompu par la suite à une époque où ces races avaient encore une importance économique non négligeable.

Recu pour publication en mai 1979.

\section{Summary \\ Colour variants in dutch Heathsheep (Heideschaap)}

A preliminary study in two Heathsheep flocks shows a biallelism at the following coat colour loci : A gouti A $\left(A^{\text {wh }}, a\right)$, Brown $B(B, b)$, Extension $E\left(E^{+}, E^{d}\right)$ and Spotting $S\left(S^{+}, S^{b}\right)$. There is perhaps another variant in $A$ gouti and another piebald allele. Compared to other so called " primitive" breeds (Icelandic, Old Norvegian or Corsican), the colour variability in Heathsheep is moderate. The basic variability may have been reduced in a first stage of evolution then the reduction process stopped for a still unknown reason.

\section{Références bibliographiques}

Adalsteinsson S., 1970. Colour inheritance in Icelandic sheep and relation between colour, fertility and fertilization. J. Agric. res. Icel., 2, 3-135.

Adalsteinsson S., WARDUM H., 1978. Frequency of color genes in Faevc Islands sheep. J. Hered., 69, 259-262.

Bemmei A. C. V. Van, 1963. Niederländische Heideschafe. Z. Säugetierk., 28, 248-255.

BERGE S., 1958. Colour in the Old Norwegian Sheep. (Norvégien, rés. angl.) Meld. Norg. Landbrukshøgsk., 37, (6) I-I 7.

Denis B., LAUVergne J. J., ThĹRET M., r978. Un variant clair du mouflon Corsico-Sarde dû à un allèle au locus $B$ (Brun). Ann. Ginét. Sél. anim., 10, 507-5I 5.

DERIBÉR̂́ M., I964. La couleur, PUF, Paris, I 28 p.

HAZEBRoEk E., I976. Veevassen en Natuurbeheer (Néer1.). Rijkinstituut. voor Natuurbeheer, Leersum.

HELDEN W. Van, MINKEMA D., I978. Inventarisatie van zeldzame Huisdierrassen im Nederland (Néerl.). Stichting zeldzame huisdierrassen, Gronnigen, 83 p. ronéoté.

Lauvergne J. J., I975. Génétique de la couleur de la toison de trois races ovines françaises : Bervichonne, Bizet et Solognote. Ann. Génét. Sél. anim., 7, 263-276.

LAUUVERGNe J. J., Adalsteinsson S., 1976. Gènes pour la couleur de la toison de la brebis Corse. Ann. Génét. Sél. anim., 8, I $53^{-1} 72$.

Lauvergne J. J., Hoogschagen P., I978. Genetic formulas for the colours in the Texel, the Dutch and the Zwartbles sheep in the Netherlands. Ann. Génét. Sél. anim., 10, 343-35I.

MASON I. L., 1969. AWold diclionary of livestock breeds. C.A.B., Farnham Royal Bucks, England.

MüLLER (sans date). Grosser mobiler Schweizer Color Atlas 254I. Chromos Verlag. Winterthur, Suisse.

Numan A., 1835. Handleiding tot de Inlandsche Schaaps-Teelt. Nederlandsche HuishondelijkeMaatschappij. Harlem, I52-I 59 .

RyDER M. L., I974. Rare sheep in the Netherlands. The Ark, 1, (6), Io.

RYDeR M. L., LAND R. B., DitchBurn, i974. Coat colour inheritance in Soay, Orkney and Shetland Sheep. J. Zool. Lond., 173, 477-485.

SEARLE A. G., I968. Comparative Genetics of Coat colour in Mammals. Logos Press, Academic Press, London and New-York, $308 \mathrm{p}$. 